\title{
Wirtschaftlichkeit kann nie losgelöst von Zielen beurteilt werden
}

Interview: Anna Sax

\section{Interaktiver
Artikel}

Wollen Sie diesen Artikel kommentieren? Nutzen Sie dafür die Kommentarfunktion in der OnlineVersion oder sehen Sie nach, was Ihre Kolleginnen und Kollegen bereits geschrieben haben: www.saez.ch/ aktuelle-ausgabe/ interaktive-beitraege/
Ende Juni findet in Basel das Forum Gesundheitswirtschaft zum Thema «Älter werden als Chance» statt. Wie lautet die zentrale Botschaft, die Sie als Programmverantwortlicher an diesen beiden Tagen vermitteln wollen?

Willy Oggier: Das Gesundheitswesen erbringt in vielerlei Hinsicht Nutzen für die Gesellschaft. Die Kosten sollten dazu ins Verhältnis gesetzt werden, getreu dem Motto «If we focus on costs, we will reduce quality. If we focus on quality, we will reduce costs». Es geht also darum, nicht nur über das Geld zu reden, sondern die Frage zu stellen, wo wir besser werden können - auch indem wir uns vergleichen. Das wollen wir vor allem mit Deutschland und Frankreich tun, doch es dürfen auch Impulse aus anderen Ländern kommen. Der Kongress ist auf Initiative von Carlo Conti, Basler Regierungsrat und Präsident der Gesundheitsdirektoren-Konferenz, entstanden.

Sie betonen den Nutzen der Gesundheitswirtschaft. Was sagen Sie den Leuten, die vor allem von einer «Kostenexplosion» sprechen?

Eine Kostenexplosion kann nur dann stattfinden, wenn die Leistungen auf gleichem Niveau bleiben. Sonst vergleicht man Äpfel mit Birnen. Wenn schon, dann müsste man von einer "Leistungsexplosion» sprechen. Und dann lautet die entscheidende gesellschaftspolitische Frage: Steht den steigenden Leistungen und Kosten ein gesellschaftlicher Mehrwert gegenüber? Darüber eine gesellschaftliche Diskussion anzustossen, ist ein Ziel unseres Forums.

Wo entsteht durch das Gesundheitswesen ein Mehrwert für die Gesellschaft?

Bei der Reduktion von potentiell verlorenen Lebensjahren, beim Gewinn von Lebensqualität, bei einem gesünderen und längeren Leben, bei der Reduktion der sozialen Diskrepanzen in der Lebenserwartung, durch die Verbesserung der Situation bestimmter älter werdender, sozial schwächerer Schichten, beispielsweise bestimmter Migrations-Gruppen. Das sind einige Stichworte dazu. In der Folge können auch Invaliditäts- und andere Sozialversicherungsrenten auf sinnvolle Weise gespart werden, und gesündere Ältere können andere wichtige Funktionen zum Beispiel in der Betreuung von Enkelkindern übernehmen.
Würden Sie das Wachstum der Gesundheitsausgaben also insgesamt als etwas Positives betrachten?

So pauschale Aussagen treffen meistens nicht zu. Und genau dies ist das Thema des Forums Gesundheitswirtschaft: Es kommt darauf an, was wir mit diesen steigenden Ausgaben erreichen und worauf wir im Gegenzug verzichten. Wenn der Bedarf der Bevölkerung steigt, was zu erwarten ist, dann kann man das Wachstum unter gewissen Umständen rechtfertigen, aber nicht einfach a priori. Die Fragestellung muss lauten, wie man dem veränderten Bedarf einer älter werdenden Gesellschaft gerecht werden kann.

Wie kann man den Nutzen des Gesundheitswesens messen?

Hier haben wir in der Schweiz grossen Nachholbedarf im Vergleich zu anderen Ländern. Deshalb wollen wir auch Vergleiche anstellen. Die Leistungserbringer müssen viel stärker sensibilisiert werden: Wo die Leistungen vor allem über Steuern und Sozialversicherungsbeiträge finanziert werden, liegt es an den Leistungserbringern, den Nutzen zu dokumentieren. Ein

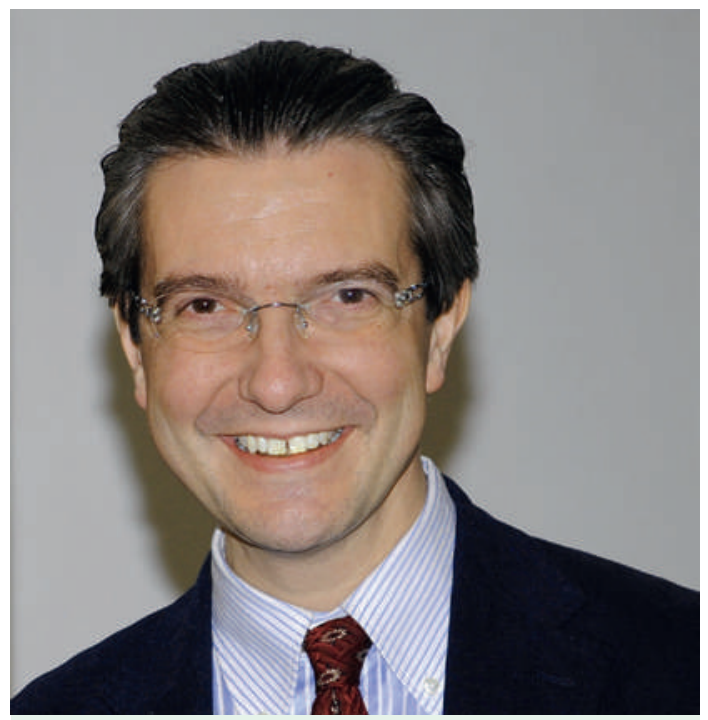

Dr. Willy Oggier ist Gesundheitsökonom und beschäftigt sich seit über 20 Jahren mit Fragen des Gesundheitswesens. Als selbständiger Berater war er in acht verschiedenen europäischen Ländern tätig.

(Foto: Peter Brandenberger) 
Beispiel ist die radikale Prostatektomie. Da gibt es zwei Dinge, die die betroffenen Männer und vielleicht auch ihre Partnerinnen vor allem interessieren, nämlich die Inkontinenz- und die Impotenzrate. Wir wissen nicht, wie das in der Schweiz aussieht. Deutsche Kliniken wie die Martiniklinik in Hamburg dokumentieren diese Outcomes seit vielen Jahren. Nochmals: Hier sind vor allem die Leistungserbringer gefordert.

Heisst das, wir wissen gar nicht, was wir erreichen mit dem, was wir machen?

Wir wissen sehr wenig. Mit der Parzellierung der Finanzierungen in der Schweiz wissen wir zudem nicht, was der Anteil des Gesundheitswesens ist und was andere krankmachende Faktoren ausmachen wie Verkehr, Bildung, Energie oder soziale Verhältnisse.

Dass die Leute länger gesund bleiben, liegt also nur zum Teil am Gesundheitssystem?

Das ist sicher richtig, wobei man hier noch stärker zielgruppenspezifisch unterscheiden könnte. Wir wissen aus ausländischen Studien, dass bestimmte Bevölkerungsschichten weniger oft zu Vorsorgeuntersuchungen gehen und unterdurchschnittliche Impfraten aufweisen. Solche Erfahrungen müssen uns zu der Frage führen: Wenn die Gesellschaft Geld investiert, wo holt sie am meisten damit heraus?

Wo soll in den kommenden Jahren investiert werden, damit der volkswirtschaftliche Nutzen der Gesundheitswirtschaft am besten zum Tragen kommt?

Es kommt darauf an, ob es sich dabei um Investitionen über Sozialversicherungen und Steuermittel handelt, oder um private Mittel. Bei letzteren ist jeder seines eigenen Glückes Schmied. Bei ersteren geht es darum, dort anzusetzen, wo gesellschaftlich am meisten Nutzen pro eingesetztem Franken erzielt werden kann. Dies ist insbesondere im Bereich der sozial schwächeren Schichten der Fall, wo wir noch immer viele Lebensjahre verlieren. Aufseiten der Leistungserbringer sind vor allem die Verbesserung des Managements von Multi-Morbiditäten, die Förderung von echten Innovationen - und damit auch die klare

\section{Forum Gesundheitswirtschaft Basel 13}

\section{Nutzen des Gesundheitswesens im Alter}

\section{Donnerstag/Freitag, 27./28. Juni 2013 im Congress Center Basel}

Die Einwohner der westeuropäischen Staaten werden immer älter, die Leistungen des Gesundheitswesens dadurch immer wichtiger - und teurer. Die steigenden Kosten haben aber auch positive Wirkungen. Am zweiten «Forum Gesundheitswirtschaft Basel» setzen sich nationale und internationale Fachleute darunter Bundesrat Alain Berset und Roche-CEO Severin Schwan - mit den medizinischen, pflegerischen, sozialen und ökonomischen Aspekten dieser Entwicklung auseinander.

Anmeldung und weitere Informationen: www.forum-gesundheitswirtschaft.ch
Absage an Schein-Innovationen - und angesichts der älter werdenden Gesellschaften die vermehrte Förderung von Psychiatrie und Rehabilitation zu erwähnen.

Müssten die Leistungen des Gesundheitswesens konsequenter Wirtschaftlichkeitsanalysen unterzogen werden?

Ich würde es nicht allein auf die Wirtschaftlichkeit beschränken. Ich würde auch sehr bewusst fragen: Was wollen wir eigentlich erreichen? Das hat viel mit den Zielen einer Gesellschaft zu tun. Wirtschaftlichkeit kann ich nie losgelöst von Zielen beurteilen.

\section{Aber in der Schweiz gibt es keine Gesundheitsziele.}

Das stimmt, aber wenn man auf kantonaler Ebene Spitalplanungen studiert oder sogar Gesundheitsleitbilder, wie sie verschiedene Kantone haben, dann lassen sich schon einige, wenigstens qualitative, Ziele formulieren. Eines zum Beispiel, das unbestritten ist, ist der Zugang zu einer guten Versorgung unabhängig vom Portemonnaie. Die Frage ist, ob sich das für jedes Tal und jedes Versorgungsgebiet und insbesondere vor dem Hintergrund der Alterung und der aussterbenden Grundversorger in 20 Jahren noch realisieren lässt. Diese Fragen müssen wir heute angehen.

Bei den Partnern des Forums Gesundheitswirtschaft sind die Pharmaindustrie und einige Privatkliniken gut vertreten. Ist es vielleicht so, dass die Gesundheitswirtschaft den Nutzen des Gesundheitswesens betont, weil sie auf Kosten der Prämien- und Steuerzahlenden höhere Gewinne erzielen will?

$\mathrm{Zu}$ den Partnern gehören auch die FMH, die Helsana und diverse öffentliche Spitäler aus den Bereichen Somatik, Psychiatrie und Geriatrie. Die Regierung des Kantons Basel-Stadt ist mit zwei Mitgliedern im Conference Board vertreten. Diese Leute lassen sich doch nicht alle vor den Karren der Industrie und der Privatkliniken spannen. Sie sind am Thema interessiert und haben wohl letztes Jahr gesehen, dass es sich lohnt, wieder mitzumachen oder wie die FMH neu einzusteigen.

Wenn Sie eine Prognose abgeben müssten: Was denken Sie, wie es den heute 50 bis 60-Jährigen in 30 Jahren gehen wird?

Naturkatastrophen und Kriege einmal ausgenommen, dürfte die Wahrscheinlichkeit hoch sein, dass ein Grossteil von ihnen weiterhin in relativ gutem Gesundheitszustand lebt. Dies hat auch damit zu tun, dass unsere Sozialsysteme stabiler sind, als uns viele Leute glauben machen wollen, und der Schweizer Souverän mit dem oft richtigen Augenmass schrittweise und vernünftige Reformen in die richtige Richtung anzuschieben vermag. Aber vielleicht ist dies auch nur Wunschdenken, auch ich bin bald fünfzig Jahre alt. 